\title{
Evaluación del ahorro de agua potable que se podrían percibir en los viveros al implementar un sistema de captación de agua de lluvia en la ciudad de Panamá
}

\section{Evaluation of the saving of drinking water that could be perceived in the nurseries when implementing a rainwater harvesting system in Panama City}

\author{
María Arévalo ${ }^{1}$, Osvaldo Martínez ${ }^{1}$, Andrea Rosales ${ }^{1}$, Maribel Pérez, Nicole Barria ${ }^{1 *}$ \\ ${ }^{1}$ Licenciatura en Ingeniería Industrial, Campus Victor Levi Sasso, Universidad Tecnológica de Panamá
}

\begin{abstract}
Resumen La escasez de agua es una problemática en incremento, que no podemos pasar por alto, porque afecta de manera negativa el desarrollo social de la población a nivel mundial. Una de las alternativas para enfrentar la falta de agua es la utilización eficiente del agua de lluvia. El objetivo principal de esta investigación fue estudiar la factibilidad del uso de un sistema de captación de agua de lluvia y cómo podría ahorrar en gran escala la cantidad de agua potable que se consume en los viveros en temporada seca en la ciudad de Panamá. Mediante la aplicación de un instrumento de recolección de datos a los encargados de los viveros estudiados (Jardines Del Bonsái S.A. y Arboretum Genera Palmarum LTD S.A.) se obtuvieron las mediciones correspondientes al consumo de agua potable en los meses de enero, febrero y marzo, con la finalidad de compararlos con el nivel de precipitaciones promedio de Panamá para esos mismos meses. Mediante los datos proporcionados por la Empresa de Transmisión Eléctrica S.A. (ETESA) se observó que los tres primeros meses del año, son los meses con menos cantidad de precipitaciones, por ende, la demanda del agua potable para el riego de plantas aumenta. Se propuso un sistema que consiste en la instalación de canales en los tejados del vivero para la recolección de agua lluvia en los últimos meses del año que son los más lluviosos. Con la implementación de este sistema se podría ahorrar más de un $2.98 \%$ del uso de agua potable en viveros durante el verano.
\end{abstract}

Palabras clave Viveros, almacenamiento, precipitaciones pluviales, captación, recolección.

\begin{abstract}
Water scarcity is a growing problem, which we cannot ignore, because it negatively affects the social development of the population worldwide. One of the alternatives to face the lack of water is the efficient use of rainwater. The main objective of this research was to study the feasibility of using a rainwater harvesting system and how it could save on a large scale the amount of drinking water consumed in dry season nurseries in Panama City. By applying a data collection instrument to the managers of the nurseries studied (Jardines Del Bonsái SA and Arboretum Genera Palmarum LTD SA), the measurements corresponding to the consumption of drinking water in the months of January, February and March were obtained, with the purpose of comparing them with the average level of rainfall in Panama for those same months. Using the data provided by the Empresa de Transmisión Eléctrica S.A. (ETESA), it was observed that the first three months of the year are the months with the least amount of rainfall, therefore, the demand for drinking water for plant irrigation increases. A system was proposed that consists of the installation of channels on the roofs of the nursery to collect rainwater in the last months of the year, which are the rainiest. With the implementation of this system, more than $2.98 \%$ could be saved from the use of drinking water in nurseries during the summer.
\end{abstract}

Keywords Nurseries, storage, rainwater, catchment, harvest, rainfall.

*Corresponding author: nicole.barria@utp.ac.pa

\section{Introducción}

La ciudad de Panamá cuenta con abundantes lluvias, de intensidad moderada a fuerte [1]. La temporada de lluvias empieza en el mes de abril y se extiende hasta diciembre, siendo los meses (septiembre, octubre y noviembre) más lluviosos. Partiendo de esta premisa, nace la idea de querer aprovechar este recurso natural, para en este caso en especial utilizarlo en beneficio del enriquecimiento y desarrollo de las plantas en temporadas secas dentro de los viveros. Este estudio ha sido apoyado con otras investigaciones que se han hecho con anterioridad a nivel mundial como lo son "La propuesta de un sistema de aprovechamiento de agua de lluvia en María Auxiliadora en Antioquia Colombia" [2], "Captación de agua de lluvia para agricultura familiar en Tlaxcala México" [3] y "Requerimientos de Infraestructura para el Aprovechamiento Sostenible del Agua Lluvia en el Campus de la Pontificia 
Barria (et al): Evaluación del ahorro de agua potable que se podrían percibir en los viveros al implementar un sistema de captación de agua de lluvia en la ciudad de Panamá

Universidad Javeriana" [4]. Se comparte de esta manera un propósito en común, el cual es darle el valor que amerita a nuestra fuente vital. Tomando en cuenta los datos recolectados se presenta un análisis en donde se trata de comprobar el objetivo de este estudio, basado en como un sistema de agua lluvia podría ahorrar a gran escala el agua potable que se utiliza para el riego de plantas en un vivero.

\section{Fundamentos teóricos}

\subsection{Conceptos generales de un vivero}

La palabra vivero proviene de latín vivarium (área generalmente cerrada, para guardar y criar plantas para observación) [1]. Un vivero es una estructura agrícola destinada a la producción de plantas, que pueden ser forestales, frutales u ornamentales. El mismo permite prever y controlar los efectos de los depredadores y patologías que dañan a las plántulas en su fase de mayor vulnerabilidad. Gracias a que se les suministran los cuidados necesarios y las condiciones adecuadas para obtener un buen desarrollo, las plantas tienen mayores oportunidades de sobrevivencia y adaptación cuando se les trasplanta a su lugar definitivo [5]. En este caso, el estudio fue enfocado en aquellos que comercializan plantas ornamentales.

\subsubsection{Tamaño del vivero}

El tamaño se define en función a la cantidad y el tipo de plantas que se producirán (por ejemplo: si la mayoría son frutales, requieren más tiempo y espacio para su desarrollo) [6].

\subsubsection{Uso del agua en un vivero}

El agua es el recurso más valioso para el funcionamiento del vivero, debido a que se requiere durante todas las etapas de producción. El vivero debe ubicarse cerca de una fuente de agua segura, como ríos, arroyos y quebradas. En lo posible, el vivero debe tener su propia fuente de agua. Debe evitarse el uso de agua potable para el consumo de la producción, como prevención a futuros conflictos y problemas en el desarrollo de las plantas [6].

\subsection{Funcionamiento general de un vivero}

El funcionamiento del vivero es mantener a las plantas en excelentes condiciones para un buen desarrollo; para que al momento que salgan del vivero tengan la suficiente energía y vigor para sobrevivir; es decir, tallo recto, raíces fuertes, hojas enteras y ser una planta sana [6].

\subsection{1 Área de captación}

Para la captación de agua lluvia se utilizan superficies como los techos de las casas y almacenes. También superficies hondas que deben estar impermeabilizadas. Otra forma de captación de agua lluvia puede ser en lagos artificiales [6].

\subsubsection{Sistema de conducción}

Es el conjunto de canales o tuberías abiertas o cerradas destinadas al transporte del agua de lluvia hasta el sistema de almacenamiento. El material tiene que ser liviano, fuerte, fácil de unir, evitando que el agua se contamine con compuestos orgánicos o inorgánicos [6].

\subsubsection{Tanques de almacenamiento}

Son estructuras modulares de diferentes materiales en donde se guarda o preserva el agua de lluvia captada. Por lo general los tanques son de forma cilíndrica y se pueden situar por encima o de manera subterránea. El tanque tiene que ser de material resistente, que evite la pérdida de agua y debe estar sellado para que no permita la entrada de polvo, insectos y contaminantes. La entrada y la salida de los tanques deben contar con mallas para la protección del agua y deben poseer dispositivos para el retiro de agua [7].

\subsection{Factores considerados que afectan el sistema de captación de agua lluvia \\ 2.3.1 Clima de Panamá}

Panamá se encuentra ubicada en el hemisferio norte latitud: entre $7^{\circ} 11^{\prime}$ Norte y $9^{\circ} 39^{\prime}$ Norte, longitud: entre $77^{\circ} 10^{\prime}$ Oeste y $83^{\circ} 03^{\prime}$ Oeste; en la zona intertropical próxima al Ecuador terrestre. La región estudiada es la región del pacífico, caracterizada en la temporada lluviosa por abundantes lluvias, acompañadas de actividad eléctrica que ocurren especialmente en horas de la tarde. La temporada lluviosa en Panamá se inicia en el mes de mayo y finaliza en noviembre, siendo septiembre y octubre los meses más lluviosos; dentro de esta temporada se presenta regularmente un período seco, entre enero y mayo [8].

\subsubsection{Sequía en Panamá}

"Los maizales están dando unos rendimientos bajísimos que distan mucho de los que se necesitan para cubrir costes de producción", así inicia la noticia del 28 de enero de 2016, del diario El Espectador [9]. El periodo de lluvia llegaba y las precipitaciones no caían, los agricultores y ganaderos no estaban preparados para una época de sequía tan pronunciada como las de pasado año.

El Niño es un fenómeno meteorológico que se presenta a nivel mundial, que muestra la variabilidad climática. Panamá no se escabulle de los efectos negativos que impactan en el sector agropecuario, en las actividades agrícolas y pecuarias. Por tal razón, se requiere estar preparados en las diferentes cadenas productivas [10], siendo este uno de los principales motores de esta investigación como una alternativa al regreso de dicho fenómeno.

\section{Metodología}

Presentaremos un modelo del diseño propuesto para la recolección de agua de lluvia que consiste en tres fases importantes. Ver figura 1. 
Barria (et al): Evaluación del ahorro de agua potable que se podrían percibir en los viveros al implementar un sistema de captación de agua de lluvia en la ciudad de Panamá

El vivero debe ubicarse cerca de una fuente de agua segura, como ríos, arroyos y quebradas En lo posible, el vivero debe tener su propia fuente de agua.

La metodología que se utilizó se basó en la selección de dos viveros de un total de 21 encontrados en la ciudad de Panamá: Vivero Jardines del Bonsái y Vivero Arboretum Genera Palmariun.

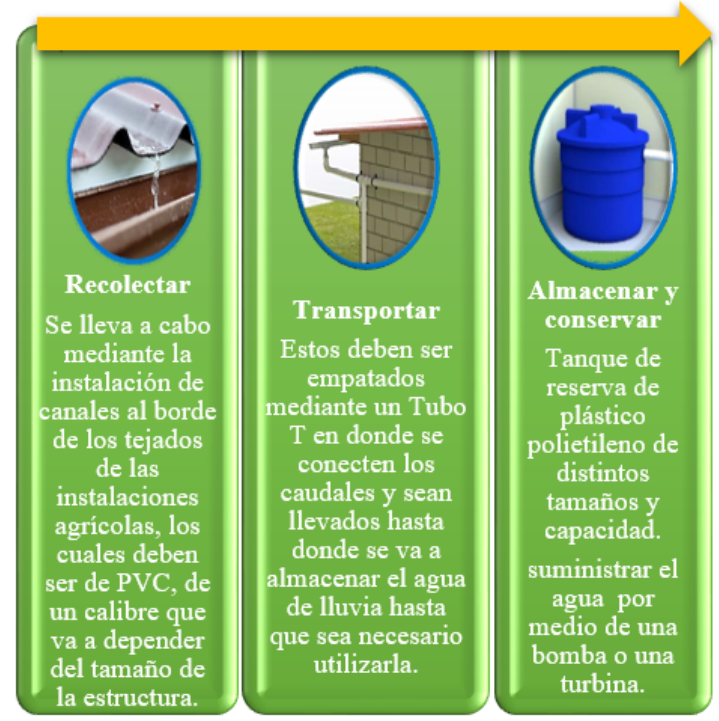

Figura 1. Funcionamiento del sistema de captación de agua lluvia.

Se procedió a visitar los viveros seleccionados, y revisar la página web de la Empresa de Transmisión Eléctrica S.A. (ETESA) que proporciona mensualmente los registros pluviales de Panamá, para luego colocar los datos en el formato de la tabla 1.

Se realizaron las conversiones de unidades correspondientes de metros cúbicos $\left(\mathrm{m}^{3}\right)$ a litros sobre metros cuadrados $\left(\frac{L}{m^{2}}\right)$ y una relación de la cantidad de agua en litros con respecto a la superficie cuadrada utilizada en el vivero, como se muestra en la siguiente ecuación (1):

$$
\mathrm{L} / \mathrm{m}^{2}=\frac{\text { agua potable utiliza por mes }\left(\mathrm{m}^{3}\right)}{\text { superficie del terreno }\left(\mathrm{m}^{2}\right)}(1000)
$$

Se investigó acerca de los meses que menos llueve en la ciudad de Panamá y se determinó que son enero, febrero y marzo, por lo cual se calculó el promedio de la demanda de agua de los viveros para dichos meses, como se muestra en la tabla 2 en unidades de litros, utilizando para la conversión la fórmula que se presenta a continuación (2):

Fórmula utilizada para calcular la demanda del agua en litros.

$$
\mathrm{DAV}=(\mathrm{DA})(\mathrm{SV})
$$

Donde:

DAV: Demanda de agua en verano (L)
DA: Demanda de agua en $\left(\frac{L}{m^{2}}\right)$

$\mathrm{SV}$ : Superficie promedio de ambos viveros $\left(\mathrm{m}^{2}\right)$

Se registraron los promedios de precipitaciones de los últimos ocho años para los meses elegidos (septiembre, octubre, noviembre, diciembre). Estos datos se utilizaron para aproximar una cantidad de agua recolectada por el sistema para abastecer el vivero durante los meses de enero, febrero, marzo.

Teniendo las precipitaciones promedio de septiembre, octubre, noviembre y diciembre se tomó en cuenta un $80 \%$ de eficiencia, tomando en cuenta que el 20\% del agua de lluvia puede ser perdida debido a salpicaduras, filtraciones entre otras cosas (UNATSABAR, 2004) [11], para un área aproximada de recolección de $40 \mathrm{~m}^{2}$ y se aproximó una cantidad de agua lluvia almacenada en esos meses (ver tabla 4).

Con los datos de la demanda en verano (enero, febrero y marzo) y la recolección de agua durante septiembre, octubre, noviembre y diciembre, se procedió a calcular con el método de la tasa de variación, se utilizó la ecuación (3), es decir el porcentaje en agua ahorrado durante los meses de verano (enero, febrero, marzo).

$$
\begin{gathered}
\text { Tasa de variación } \%=\frac{\text { DAV-AR }}{\text { DVA }}(100) \\
\% \text { de agua potable }=100-\%
\end{gathered}
$$

Donde:

$\%$ : porcentaje de agua potable utilizada

DAV: Demanda de agua en verano (L)

AR4: Agua recolectada en los últimos 4 meses del año

(L)

\section{Resultados}

La tabla 1 muestra los datos recolectados con la aplicación del instrumento a los encargados de los viveros, al igual que el nivel de precipitaciones proporcionado por ETESA [12] estación meteorológica de Albrook.

Tabla 1. Datos de precipitaciones y cantidad de agua utilizada en el vivero

\begin{tabular}{|l|l|l|l|}
\hline \multirow{4}{*}{ Meses } & $\begin{array}{l}\text { Precipitaciones } \\
\text { (Estación de } \\
\text { Albrook) }\end{array}$ & $\begin{array}{l}\text { Vivero Jardines } \\
\text { del Bonsái }\end{array}$ & $\begin{array}{l}\text { Vivero } \\
\text { Arboretum } \\
\text { Genera } \\
\text { Palmariun }\end{array}$ \\
\cline { 2 - 5 } & $\begin{array}{l}\text { Precipitaciones } \\
\text { por mes } \\
\left(\mathrm{L} / \mathrm{m}^{2}\right)\end{array}$ & $\begin{array}{l}\text { Cantidad de agua } \\
\text { utilizada al mes } \\
\left(\mathrm{m}^{3}\right)\end{array}$ & $\begin{array}{l}\text { Cantidad de agua } \\
\text { utilizada al mes } \\
\left(\mathrm{m}^{3}\right)\end{array}$ \\
\hline Enero & 77.2 & 265 & 286.2 \\
\hline Febrero & 1.7 & 280 & 302.4 \\
\hline Marzo & 1.8 & 285 & 307.8 \\
\hline Abril & 200.1 & 270 & 291.6 \\
\hline Mayo & 160.5 & 85 & 91.8 \\
\hline
\end{tabular}


Barria (et al): Evaluación del ahorro de agua potable que se podrían percibir en los viveros al implementar un sistema de captación de agua de lluvia en la ciudad de Panamá

La siguiente tabla muestra los datos proporcionados por los dos viveros de la muestra, de unidades cubicas (m3) a L/m2 (1).

Tabla 2. Datos de precipitaciones y cantidad de agua utilizada en el vivero en $(\mathrm{L} / \mathrm{m} 2)$

\begin{tabular}{|l|l|l|l|l|}
\hline Meses & $\begin{array}{l}\text { Precipitaciones } \\
\text { por mes } \\
\left(\mathbf{L} / \mathbf{m}^{2}\right)\end{array}$ & $\begin{array}{l}\text { Cantidad } \\
\text { de agua } \\
\text { utilizada } \\
\text { al mes } \\
\left(\mathbf{L} / \mathbf{m}^{2}\right)\end{array}$ & $\begin{array}{l}\text { Cantidad agua } \\
\text { de } \\
\text { utilizada } \\
\text { al mes } \\
\left(\mathbf{L} / \mathbf{m}^{2}\right)\end{array}$ & $\begin{array}{l}\text { Promedio de } \\
\text { agua potable } \\
\text { utilizada en } \\
\text { ambos } \\
\text { viveros }\left(\mathbf{L} / \mathbf{m}^{2}\right)\end{array}$ \\
\hline Enero & 77.2 & 66.25 & 81.77 & 74.01 \\
\hline Febrero & 1.7 & 70.00 & 86.40 & 78.20 \\
\hline Marzo & 1.8 & 71.25 & 87.94 & 79.60 \\
\hline Abril & 200.1 & 67.50 & 83.31 & 75.41 \\
\hline Mayo & 160.5 & 21.25 & 26.23 & 23.74 \\
\hline
\end{tabular}

Tomando en cuenta que según las estadísticas de precipitaciones proporcionadas por ETESA [12]: Enero, febrero y marzo son los meses en que menos llueve, la demanda de agua potable en los viveros incrementa "(ver gráfica 1 y 2)" y en base a esto se puede concluir que los meses ideales para recolectar agua lluvia son septiembre, octubre, noviembre y diciembre (ver gráfica 2), para utilizar la misma durante el verano del siguiente año.

Por lo que se puede estimar una demanda de agua utilizada en veranos por los viveros (ver tabla 3 ).

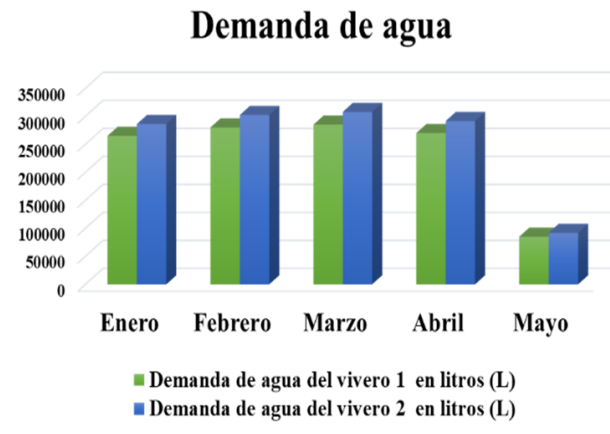

Gráfica 1. Precipitaciones para enero, febrero y marzo vs la demanda de agua en esos mismos meses de cada vivero.

Tabla 3. Total de la demanda de agua en verano (enero, febrero, marzo)

\begin{tabular}{|l|l|l|l|}
\hline Meses & $\begin{array}{l}\text { Cantidad de } \\
\text { agua utilizada al } \\
\text { mes }\left(\mathbf{L} / \mathbf{m}^{2}\right)\end{array}$ & $\begin{array}{l}\text { Cantidad de } \\
\text { agua utilizada al } \\
\text { mes }\left(\mathbf{L} / \mathbf{m}^{2}\right)\end{array}$ & $\begin{array}{l}\text { Promedio de } \\
\text { agua potable } \\
\text { utilizada en } \\
\text { ambos viveros }\end{array}$ \\
\hline Enero & 66.25 & 81.77 & 74.01 \\
\hline Febrero & 70.00 & 86.40 & 78.20 \\
\hline Marzo & 71.25 & 87.94 & 79.60 \\
\hline $\begin{array}{l}\text { Total de } \\
\text { agua } \\
\text { utilizada } \\
\text { en } \\
\text { verano: }\end{array}$ & & & \\
\hline & & & $\mathbf{2 3 1 . 8 1}\left(\mathrm{L} / \mathrm{m}^{2}\right)$ \\
\hline
\end{tabular}

El promedio total de agua que se necesitaría para cubrir con la demanda del verano seria de $231.81 \mathrm{~L} / \mathrm{m}^{2}$, por ende, la cantidad de agua recolectada debe ser en base a este dato.

En la tabla 4 se muestra el promedio de precipitaciones de los meses de septiembre, octubre, noviembre y diciembre de los últimos ocho años, siendo estos los meses con mayor cantidad de precipitaciones a lo largo del año para la captación del agua lluvia, ver gráfica 2.

Conforme a las estadísticas proporcionadas por ETESA [12], la cantidad promedio de precipitaciones desde septiembre a diciembre es de $810.38 \mathrm{~L} / \mathrm{m}^{2}$.

En la tabla 5 y la gráfica 2 muestran las precipitaciones promedio por mes y la cantidad de agua recolectada con un $80 \%$ de eficiencia.

Tabla 4. Precipitación promedio total en los últimos meses del año

\begin{tabular}{|l|l|}
\hline Meses & Precipitaciones por mes $\left(\mathbf{L} / \mathbf{m}^{\mathbf{2}}\right)$ \\
\hline Septiembre & 194.3625 \\
\hline Octubre & 237.3375 \\
\hline Noviembre & 199.25 \\
\hline Diciembre & 179.4285 \\
\hline Total: & $\mathbf{8 1 0 . 3 8}$ \\
\hline
\end{tabular}

Porcentaje de agua que se podría recolectar en $40 \mathrm{~m}^{2}$ de techo con un $80 \%$ de eficiencia.

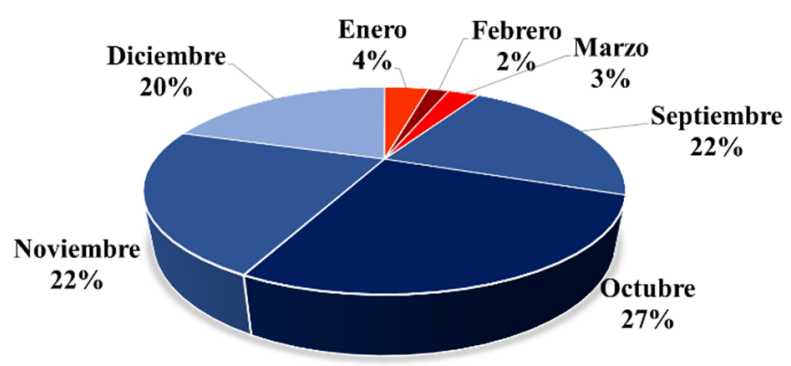

Gráfica 2. Precipitaciones para septiembre, octubre, noviembre y diciembre (considerados los meses aptos para la captación de agua lluvia).

De acuerdo a los resultados de la tabla 5 se concluyó que durante los últimos cuatro meses del año se podrían recolectar aproximadamente $25932.11 \mathrm{~L}$ de agua para ser utilizados durante el verano.

Tabla 5: Precipitación promedio total en los últimos meses del año

\begin{tabular}{|c|c|c|c|}
\hline Meses & $\begin{array}{l}\text { Precipitaciones } \\
\text { por mes }\left(\mathrm{L} / \mathrm{m}^{2}\right)\end{array}$ & $\begin{array}{l}\text { Agua } \\
\text { recolectada en } \\
\text { litros (L) en un } \\
\text { techo de } 40 \mathrm{~m}^{2}\end{array}$ & $\begin{array}{l}\text { Techo de } 40 \mathrm{~m}^{2} \text { de } \\
\text { captación con } \\
80 \% \text { de eficiencia } \\
\text { (L) }\end{array}$ \\
\hline Septiembre & 194.36 & 7774.50 & 6219.60 \\
\hline Octubre & 237.34 & 9493.50 & 7594.80 \\
\hline Noviembre & 199.25 & 7970.00 & 6376.00 \\
\hline Diciembre & 179.43 & 7177.14 & 5741.71 \\
\hline Total: & & & 25932.11 L \\
\hline
\end{tabular}


Barria (et al): Evaluación del ahorro de agua potable que se podrían percibir en los viveros al implementar un sistema de captación de agua de lluvia en la ciudad de Panamá

Cambiamos la demanda de agua en verano (DAV) a unidades de litros (L).

$$
\mathrm{DAV}=231.81 \mathrm{~L} / \mathrm{m}^{2}\left(3750 \mathrm{~m}^{2}\right)=869,287.5 \mathrm{~L}
$$

Con los datos de la demanda de agua en veranos (DAV) y el agua recolectada en los últimos cuatro meses del año (AR4) la tasa de variación encontrada es:

$$
\text { Tasa de variación } \%=\frac{869,287.5 \mathrm{~L}-25,932.11 \mathrm{~L}}{869,287.5 \mathrm{~L}} \times 100
$$

$\%=97.02$ de agua potable utilizada

$\%$ de ahorro de agua potable $=100-97.02$

$\%$ de ahorro de agua potable $=\mathbf{2 . 9 8}$

Con un sistema de captación de agua de lluvia se ahorrarían aproximadamente $2.98 \%$ de agua potable durante todo el verano (enero, febrero, marzo) [11-12].

\section{Conclusiones}

- De acuerdo con los resultados obtenidos en el análisis de los datos, se determinó que el porcentaje de agua potable que se ahorra no es significativo, en comparación a la cantidad promedio de agua que requieren las plantas en temporadas secas.

- Se concluyó que la idea de ahorrar agua potable ha generado un gran interés por parte de los dueños de los viveros, por el impacto positivo que esto podría representar para la naturaleza si se realiza a una mayor escala de reciclaje de agua de lluvia. Por ende, apoyando esta premisa, la idea de los canales de tubo de PVC para la recolección de agua de lluvia en los techos no resultó ser la mejor alternativa, pero otras alternativas como la creación de lagos artificiales, represas u otras estructuras a fines, quedan a expensas de investigación, ampliando el panorama de posibilidades de contribuir a un mejor cuidado del ambiente y a un mejor aprovechamiento de los recursos naturales que nos proporciona la madre tierra.

- El ahorro de agua potable para una superficie promedio de $3750 \mathrm{~m}^{2}$ poblada de plantas de acuerdo al estudio realizado es de $2.98 \%$. Este porcentaje puede crecer, estudiando e investigando otras alternativas como las antes mencionadas

\section{AGRADECIMIENTOS}

\section{A Dios}

Por permitirnos llevar nuestra investigación con éxito y poder culminarla de la misma manera.

\section{A nuestra profesora asesora, Nicole Barría}

Por siempre tener críticas constructivas y buscar la mejora de nuestra investigación con el fin de enriquecer nuestros conocimientos.

\section{A nuestros padres}

Por tener siempre las palabras de aliento en el momento indicado, para seguir siendo nuestros polos a tierra y a la vez nuestros propulsores para triunfar.

\section{A nosotros}

Por formar un grupo de trabajo sólido y nutrido, ayudándonos y cumpliendo con nuestros objetivos.

\section{Al personal de los viveros}

Por brindarnos una grata atención y responder todas nuestras interrogantes acerca del proceso del cuidado de las plantas.

\section{REFERENCIAS}

[1] https://es.wikipedia.org/wiki/Clima_de_Panam\%C3\%A1

[2] N. Palacios. (2010) "PROPUESTA DE UN SISTEMA DE Aprovechamiento de agua lluvia, como alternativa para el ahorro de agua potable, en la institución educativa maría auxiliadora de Caldas, Antioquia”. Disponible: http://tesis.udea.edu.co/bitstream/10495/1325/1/PropuestaSistem aAprovechamientoAguaLluvia.pdf

[3] A. Quispe. (2005) Captación de agua de lluvia para la agricultura familiar, una experiencia en comunidades rurales de Tlaxcala. Disponible:

http://132.248.9.34/hevila/Boletindelarchivohistoricodelagua/20 08/vol13/noesp/9.pdf

[4] J. L. Estupiñán. H. O. Zapata. (diciembre 2010) Requerimientos de Infraestructura para el Aprovechamiento Sostenible del Agua Lluvia en el Campus de la Pontificia Universidad Javeriana. https://repository.javeriana.edu.co/bitstream/handle/10554/1265/ ing05.pdf?sequence $=2$

[5]C. Yanes, A. Orozco, M. Rojas, M. Sánchez, V. Cervantes. (1997). La reproducción de las plantas: semillas y meristemos. (1 edición) Disponible:

(http://bibliotecadigital.ilce.edu.mx/sites/ciencia/volumen3/cienc ia3/157/htm/sec_7.htm

[6] L. Chandeck, G. González, R. Orozco, R. Beltrán, A. León, J. Pimentel, N. Karina, A. Morales, C. Arauz, B. Alzamora, Manual De Viveros Forestales, (mayo 2012) Autoridad Nacional del Ambiente, ANAM. Disponible: https://www.mida.gob.pa/upload/documentos/librosdigitales/PID CAC/Manual_Miveros_Forestales/manual_viveros_forestales.pd $\mathrm{f}$

[7] Captación de agua de lluvia, Soluciones Hidropluviales (2012). Disponible: http://hidropluviales.com/2012/11/29/captacionagua-de-lluvia/

[8] Hidromet, ETESA (2009), Factores que determinan el clima en Panamá. Disponible: http://www.hidromet.com.pa/clima_panama.php

[9] EFE (2016, enero 28), Cultivos de maíz en Panamá están desapareciendo por las altas temperaturas, El Espectador. Disponible: https://www.elespectador.com/noticias/medioambiente/cultivos-de-maiz-panama-estan-desapareciendo-altastemp-articulo-613422 
Barria (et al): Evaluación del ahorro de agua potable que se podrían percibir en los viveros al implementar un sistema de captación de agua de lluvia en la ciudad de Panamá

[10] IDIAP Panamá (2015, Mayo 22), Alternativas tecnológicas generadas por el IDIAP para afrontar problemas de sequía. Disponible: http://www.idiap.gob.pa/2015/05/22/alternativastecnologicas-generadas-por-el-idiap-para-afrontar-problemas-desequia/

[11]O, Vargas. I. González, N. Y Trujillo, J. M. (2018). Análisis de un sistema de cosecha de agua a pequeña escala con finalidad pecuaria. Revista Luna Azul, 46, 82-101. Recuperado de http://200.21.104.25/lunazul/index.php/component/content/articl e?id=270. DOI: 10.17151/luaz.2018.46.3

[12]ETESA (2018), Datos diarios. Disponible: http://www.hidromet.com.pa/datos_diarios.php 